\title{
Uses of Ex-Vivo Lung Perfusion in Pulmonary Translational Medicine
}

\author{
Patrick G Chan*1, Ernest G Chan ${ }^{2}$ and Pablo G Sanchez ${ }^{3}$ \\ Department of Cardiothoracic Surgery, USA \\ *Corresponding author: Patrick G Chan, Department of Cardiothoracic Surgery, University of Pittsburgh, Pittsburgh, \\ Pennsylvania, USA
}

\begin{tabular}{|c|c|}
\hline ARTICLE INFO & ABSTRACT \\
\hline Received: 幽 October 28, 2019 & Abbreviations: MSC: Mesenchymal Stromal Cells; MAPC: Multipotent Adult Progenitor \\
\hline Published: November 08, 2019 & $\begin{array}{l}\text { Cells; LLL: Left Lower Lobes; RLL: Right Lower Lobes; BAL: Bronchoalveolar Lavage } \\
\text { Fluid; NO: Nitric Oxide }\end{array}$ \\
\hline
\end{tabular}

Citation: Patrick G Chan, Ernest G Chan, Pablo G Sanchez. Uses of Ex-Vivo Lung Perfusion in Pulmonary Translational Medicine. Biomed J Sci \& Tech Res 22(4)2019. BJSTR. MS.ID.003781.

\section{Introduction}

End-stage lung disease is treated with lung transplantation. The advancements in lung transplantation has allowed it to be applied to a greater number of pulmonary etiologies [1,2]. Unlike other solid organs such as kidney and liver, only $15-25 \%$ of lungs from donors are transplanted [3]. These low percentages are a testament to the delicacy of lungs. Because of the difficulty of obtaining lungs suitable for transplantation, multiple methods have been used to expand the donor pool. The recent advancement of ex-vivo lung perfusion (EVLP) has arguably the greatest impact on revolutionizing the evaluation of donor lungs and expanding the number of lungs suitable for transplantation. EVLP allows for lungs to be ventilated, oxygenated and perfused for assessment prior to implantation. By creating a physiologic and controlled milieu, EVLP can also be used as platform for reconditioning and repairing lungs [4-9]. In the realm of lung transplantation, there have been promising results in drug, gene, stem cell and medical gas therapy. In addition, EVLP can be applied to thoracic oncology and regenerative medicine.

\section{Drug Therapy}

Using the EVLP circuit, medications can be added in the pulmonary vessels and bronchus for directed therapy in study lungs. In the case of donor lungs, this would avoid systemic exposure and toxicity. One prime example of directed drug therapy for treatment of donor lungs is in the case of pulmonary emboli, which is a contraindication for transplantation. Machuca and colleagues described a case in which a pulmonary embolism was causing hemodynamic compromise in the donor lungs. Using EVLP, directed thrombolysis with alteplase was done followed by multiple flushings to wash out any residual emboli [10]. Another contraindication for transplantation of donor lungs is pulmonary infections, whether it is associated with mechanical ventilation or aspiration. Using an EVLP, Nakajima and colleagues were able to show that high-dose antimicrobial agents added to the perfusates of lungs can significantly decrease microbial load [11]. Similarly, after a severe Pseudomonas aeruginosa infection was induced in the lungs of pigs, Zinne et al. placed these lungs on an EVLP circuit with added colistin to the perfusate. These lungs were kept on the circuit for treatment and re-implanted into the pigs. In the EVLP group, there was reduced overall mortality compared to conventional systemic intravenous antibiotic treatment of the pneumonia (control). The symptoms of infection were also less severe in the EVLP group. Therefore, though more research needs to be done, this could be an avenue of increasing the donor pool of lungs [12].

\section{Gene Therapy}

Much like drug therapy, directed gene therapy can also be done on the EVLP circuit. Anti-inflammatory cytokines can be used to treat donor lungs. Specifically, IL-10, which inactivates 
antigen-presenting cells and inhibits the proinflammatory cytokine cascade, has been studied as gene therapy in EVLP. Machuca et al. [13] showed that treating lungs on EVLP with IL-10 transfected adenovirus is safe and improves post-transplant lung function and outcomes over EVLP alone in a porcine model [14]. This translated to a human model, as lungs from 10 donors deemed unusable for transplantation were treated with IL-10 gene therapy while on EVLP. Not only did these lungs had a more favorable antiinflammatory cytokine profile, these lungs showed improvement in arterial oxygen pressure and pulmonary vascular resistance compared to the controls [14].

\section{Stem-Cell Therapy}

The interplay between pro- vs. anti-inflammatory cytokines and cellular profiles comes into significant play in transplantation. Studies have shown that mesenchymal stromal cells (MSC) or multipotent adult progenitor cells (MAPC) can affect this balance $[15,16]$. Using EVLP, Nakajima and colleagues delivered MSCs to the pulmonary artery oflungs and showed that apoptosis was decreased compared to control. Acute lung injury and pro-inflammatory markers were also decreased in the MSC group. Air movement was also improved, as peak airway pressures were decreased in the MSC group compared to control as well [17]. It has been hypothesized that MAPCs can decreased primary graft dysfunction in transplanted organs by decreasing ischemic-perfusion injury. La Francesca and colleagues bronchoscopically instilled MAPCs into the left lower lobes (LLL) and vehicle control into the right lower lobes (RLL) of donor lungs. The lungs were placed into the EVLP circuit and perfused and ventilated. After the EVLP run, the lungs were assessed for histologic injury and for inflammatory markers in bronchoalveolar lavage fluid (BAL) and lung tissue. La Francesca and colleagues showed that the LLLs treated with MAPCs had significantly decreased histological inflammation and decreased inflammatory markers compared to the RLLs [18].

\section{Medical Gas Therapy}

Various gas therapies can also be used to study lungs on EVLP. Inhaled nitric oxide (NO) is used quite frequently in the realm of lung transplantation as a pulmonary vasodilator $[19,20]$. There have been some discussions whether pre-transplant NOventilation could be helpful during the post-operative course. In a rat model, Dong and colleagues, ventilated rat lung with NO while on EVLP. They were able to show that lungs with NO-ventilation had significantly reduced wet:dry weight ratio, better oxygenation, and reduced pulmonary vascular resistance. There was also a maintenance of endothelial NO synthase (eNOS) and a better antiinflammatory profile for lungs ventilated with NO [21].

\section{EVLP Outside of Lung Transplantation}

\section{Thoracic Oncology}

In 1958, Creech and colleagues used isolated lung perfusion with chemotherapeutic agents to treat pulmonary malignancies on an extracorporeal circuit much like EVLP. By using an EVLP circuit, lungs stricken with cancer can be given higher doses of chemotherapy without exposing the rest of the body to toxic levels of chemotherapy. This was successfully shown by Weksler and colleagues. They showed that in-vivo isolated single lung perfusion in rats is feasible with low mortality and morbidity [22]. Reck dos Santos and colleagues used this concept in the setting of pulmonary metastases. Reck dos Santos cannulated the pulmonary artery and veins in pigs in vivo, with a circuit similar to EVLP. The left lung was perfused for 4 hours with doxorubicin. There was no systemic exposure to doxorubicin with homogeneously distribution in the lung. There was also no evidence of acute lung injury [23]. Though promising, further studies are needed to determine is long term efficacy on local recurrence, toxicity, pulmonary function and overall survival.

\section{Regenerative Medicine and Bioengineering}

Organ engineering is another method to increase the number of organs available for patients with end-stage lung disease. Ott and colleagues developed techniques to decellularize lungs from various animals. These decellularized lungs are left with extracellular matrix and architecture that serves as a scaffold for cellular deposition. Ott was able to generate functional pulmonary vasculature by repopulating the vascular beds with endothelial and perivascular cells [24,25]. Ultimately, this decellularized platform can be used to potentially "grow" lungs for possible transplantation. EVLP has been suggested as a bioreactor circuit for delivery of cells to for the artificial organ and for providing a physiological environment for proliferation. It can also then be used as an assessment tool for artificial lungs.

\section{Conclusion}

Currently, EVLP is predominantly used as a platform for assessment of marginal donor lungs. With the capabilities of EVLP to ventilate and perfuse lungs, it can be used as a potent platform for lung therapies in and outside the realm of lung transplantation. Marginal lungs can be treated on the EVLP circuit for optimization prior to transplantation. Meanwhile, the EVLP or circuits similar can be used for lung isolation for oncologic treatments, or as a bioreactor platform for the growth of artificial organs.

\section{References}

1. Chan EY, Goodarzi A, Sinha N, Nguyen DT, Youssef JG, et al. (2018) Long-term survival in bilateral lung transplantation for sclerodermarelated lung disease. The Annals of thoracic surgery 105(3): 893-900.

2. Chaparro C, Keshavjee S (2016) Lung transplantation for cystic fibrosis: An update. Expert Rev Respir Med 10(12): 1269-1280.

3. Pomfret EA, Sung RS, Allan J, Kinkhabwala M, Melancon JK (2008) Solving the organ shortage crisis: The 7th annual american society of transplant surgeons' state-of-the-art winter symposium. American journal of transplantation : official journal of the American Society of Transplantation and the American Society of Transplant Surgeons 8(4): 745-752.

4. Cypel M, Yeung JC, Hirayama S (2008) Technique for prolonged normothermic ex vivo lung perfusion. The Journal of heart and lung transplantation: the official publication of the International Society for Heart Transplantation 27(12): 1319-1325. 
5. Cypel M, Yeung JC, Liu M (2011) Normothermic ex vivo lung perfusion in clinical lung transplantation. The New England journal of medicine 364(15): 1431-1440.

6. Cypel M, Yeung JC, Machuca T (2012) Experience with the first 50 ex vivo lung perfusions in clinical transplantation. The Journal of thoracic and cardiovascular surgery 144(5): 1200-1206.

7. Hardesty RL, Griffith BP (1987) Autoperfusion of the heart and lungs for preservation during distant procurement. The Journal of thoracic and cardiovascular surgery 93(1): 11-18

8. Sanchez PG, Davis RD, D'Ovidio F (2014) The novel lung trial oneyear outcomes. The Journal of Heart and Lung Transplantation 33(4) S71-S72.

9. Steen S, Sjoberg T, Pierre L, Liao Q, Eriksson L (2001) Transplantation of lungs from a non-heart-beating donor. Lancet (London, England) 357(9259): 825-829.

10. Machuca TN, Hsin MK, Ott HC (2013) Injury-specific ex vivo treatment of the donor lung: Pulmonary thrombolysis followed by successful lung transplantation. Am J Respir Crit Care Med 188(7): 878-880.

11. Nakajima D, Cypel M, Bonato R (2016) Ex vivo perfusion treatment of infection in human donor lungs. American journal of transplantation: official journal of the American Society of Transplantation and the American Society of Transplant Surgeons 16(4): 1229-1237.

12. Zinne N, Krueger M, Hoeltig D (2018) Treatment of infected lungs by ex vivo perfusion with high dose antibiotics and autotransplantation: A pilot study in pigs. PloS one 13(3): e0193168.

13. Machuca TN, Cypel M, Bonato R (2017) Safety and efficacy of ex vivo donor lung adenoviral il-10 gene therapy in a large animal lung transplant survival model. Hum Gene Ther 28(9): 757-765.

14. Cypel M, Liu M, Rubacha M (2009) Functional repair of human donor lungs by il-10 gene therapy. Sci Transl Med 1(4): 4ra9.

15. De Paul MA, Palmer M, Lang BT (2015) Intravenous multipotent adult progenitor cell treatment decreases inflammation leading to functional recovery following spinal cord injury. Sci Rep 5: 16795.

\section{ISSN: 2574-1241}

DOI: 10.26717/BJSTR.2019.22.003781

Patrick G Chan. Biomed J Sci \& Tech Res

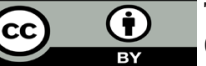

This work is licensed under Creative Commons Attribution 4.0 License

Submission Link: https://biomedres.us/submit-manuscript.php
16. Iyer SS, Rojas M (2008) Anti-inflammatory effects of mesenchymal stem cells: Novel concept for future therapies. Expert Opin Biol Ther 8(5): 569-581.

17. Nakajima D, Watanabe Y, Ohsumi A (2017) Mesenchymal stem cell therapy for ex vivo repair of ischemic injury in pig donor lungs. The Journal of Heart and Lung Transplantation 36(4): S68.

18. La Francesca S, Ting AE, Sakamoto J (2014) Multipotent adult progenitor cells decrease cold ischemic injury in ex vivo perfused human lungs: An initial pilot and feasibility study. Transplantation research 3(1): 19.

19. Pasero D, Martin EL, Davi A, Mascia L, Rinaldi M (2010) The effects of inhaled nitric oxide after lung transplantation. Minerva anestesiologica 76(5): 353-361.

20. Yerebakan C, Ugurlucan M, Bayraktar S, Bethea BT, Conte JV (2009) Effects of inhaled nitric oxide following lung transplantation. Journal of cardiac surgery 24(3): 269-274.

21. Dong BM, Abano JB, Egan TM (2009) Nitric oxide ventilation of rat lungs from non-heart-beating donors improves posttransplant function. American journal of transplantation: official journal of the American Society of Transplantation and the American Society of Transplant Surgeons 9(12): 2707-2715.

22. Weksler B, Schneider A, Ng B, Burt M (1993) Isolated single lung perfusion in the rat: An experimental model. Journal of applied physiology (Bethesda, Md: 1985) 74(6): 2736-2739.

23. Reck Dos Santos P, Sakamoto J, Chen M (2016) Modified in vivo lung perfusion for local chemotherapy: A preclinical study with doxorubicin The Annals of thoracic surgery 101(6): 2132-2140.

24. Gilpin SE, Guyette JP, Gonzalez G (2014) Perfusion decellularization of human and porcine lungs: Bringing the matrix to clinical scale. The Journal of heart and lung transplantation: the official publication of the International Society for Heart Transplantation 33(3): 298-308.

25. Gilpin SE, Ott HC (2015) Using nature's platform to engineer bioartificial lungs. Ann Am Thorac Soc 12 Suppl 1: S45-49.

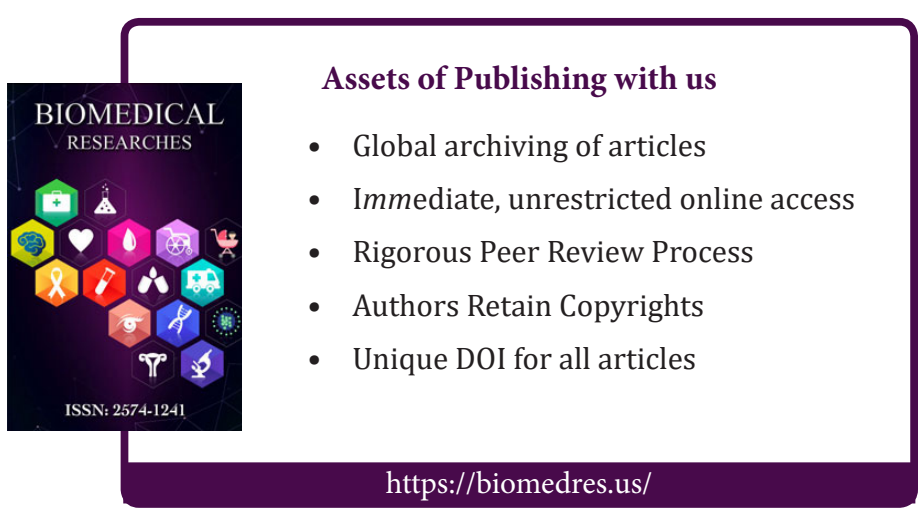

